\title{
Attention affects the stereoscopic depth aftereffect
}

\section{David Rose, Mark F Bradshaw, Paul B Hibbard}

Department of Psychology, University of Surrey, Guildford GU2 7XH, UK; e-mail: d.rose@surrey.ac.uk Received 20 December 2001, in revised form 28 January 2003; published online 14 May 2003

\begin{abstract}
Preattentive' vision is typically considered to include several low-level processes, including the perception of depth from binocular disparity and motion parallax. However, doubt was cast on this model when it was shown that a secondary attentional task can modulate the motion aftereffect (Chaudhuri, 1990 Nature 344 60-62). Here we investigate whether attention can also affect the depth aftereffect (Blakemore and Julesz, 1971 Science 171 286-288). Subjects adapted to stationary or moving random-dot patterns segmented into depth planes while attention was manipulated with a secondary task (character processing at parametrically varied rates). We found that the duration of the depth aftereffect can be affected by attentional manipulations, and both its duration and that of the motion aftereffect varied with the difficulty of the secondary task. The results are discussed in the context of dynamic feedback models of vision, and support the penetrability of low-level sensory processes by attentional mechanisms.
\end{abstract}

\section{Introduction}

There has recently been a change in our understanding of selective attention to perceptual stimuli (Driver and Frackowiak 2001; Bradshaw and Rose 2002; Paradiso 2002). In place of the previous model, of 'preattentive' processing followed by a selective filtering mechanism, influences of attention on 'early' vision have recently been revealed in several ways. These include psychophysical demonstrations of changes in the basic analysis of motion (eg Chaudhuri 1990; Shulman 1993; Culham and Cavanagh 1994; Lankheet and Verstraten 1995; Rees et al 1997; von Grünau et al 1998; Ho 1998; Watanabe and Shimojo 1998; Alais and Blake 1999; Georgiades and Harris 2000a, 2000b, 2002; Gray 2000; Nishida and Ashida 2000; Raymond 2000; Krauzlis and Adler 2001), orientation (Spivey and Spirn 2000), texture segmentation (Yeshurun and Carrasco 2000), and stimulus detection (Plainis et al 2001; Cameron et al 2002); see also most of the papers in Spekreijse (2000). Effects of attention have also been shown neurophysiologically in monkeys (eg Motter 1993; Vidyasagar 1998; Posner and Gilbert 1999; Mehta et al 2000a, 2000b; Vanduffel et al 2000). Perhaps the most relevant demonstrations reveal attentional alterations as early as V1 itself in humans, by direct monitoring of V1 activity (eg Rees et al 1997; Büchel et al 1998; Watanabe et al 1998a, 1998b; Posner and Gilbert 1999; Smith et al 2000; Martínez et al 2001; Olson et al 2001).

Numerous theorists have suggested it is the feedback connections from higher centres which mediate such effects (eg Deco and Schürmann 2000; Lamme and Roelfsema 2000; Lamme and Spekreijse 2000; Spivey and Spirn 2000; Suder and Wörgötter 2000; Schroeder et al 2001). However, most sensory processes engage several structures at multiple stages, and it remains to be demonstrated empirically whether all the processes mediated by early structures can be affected by attention, and how selective those attentional effects are.

Here, we investigate whether attention can affect the duration of the depth aftereffect tested with random-dot stereograms (RDSs) (Blakemore and Julesz 1971; Rose and Price 1995). The extraction of depth from RDSs is known to depend at least in part on early cortex (Poggio 1995; Trotter et al 1996; Prince et al 2000; von der Heydt et al 2000). 
We use a well-established psychophysical technique which was developed on the motion aftereffect (Chaudhuri 1990; Rees et al 1997; Georgiades and Harris 2000a, 2000b, 2002; Nishida and Ashida 2000), and in which attention is capable of affecting even prebinocular channels (Nishida and Ashida 2000), to study both depth and motion aftereffects.

\section{Method}

Stimuli were generated on an Apple Macintosh computer and displayed on two Apple monochrome monitors $(67 \mathrm{~Hz}$ refresh rate) viewed through a mirror stereoscope. The displays resembled those used by Blakemore and Julesz (1971), as shown schematically in figure 1. All displays contained a $5.8 \mathrm{deg}$ wide square background of $1.25 \mathrm{~min}$ of arc pixels, each pixel having a random $50 \%$ chance of being bright or dark. The mean luminance was $33 \mathrm{~cd} \mathrm{~m}^{-2}$ and the rest of the visual field was dark. For the baseline conditions, two $2.1 \mathrm{deg}$ wide square patches of random dots stood out by $7.5 \mathrm{~min}$ disparity in front of the background. The patches were symmetrically placed $(0.6 \mathrm{deg}$ nearest edge distance) above and below a 0.4 deg wide square window in the centre of the display and in the same depth plane. The window was black and contained a white fixation cross. The display was viewed through a mirror stereoscope at $97 \mathrm{~cm}$ distance.

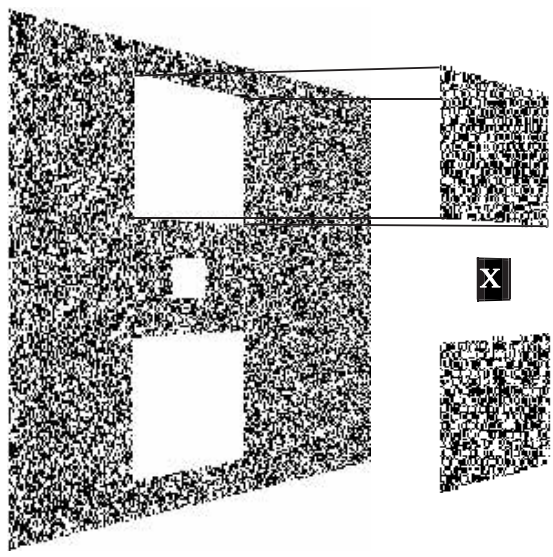

(a)

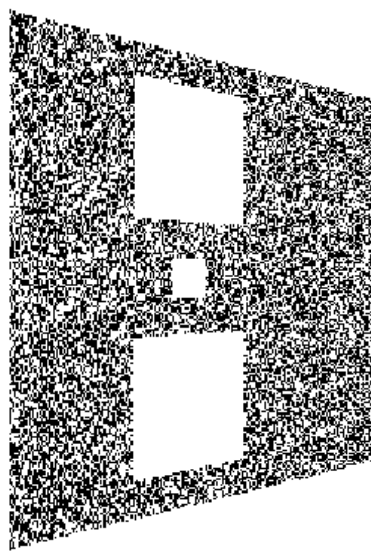

(b)

Figure 1. (a) Diagram of stimulus display in baseline condition (not to scale). (b) Stimulus display with unequal depths of the upper and lower patches.

For depth aftereffects the subject began by viewing the baseline display (figure 1a) for $15 \mathrm{~s}$. Adaptation then took place for $30 \mathrm{~s}$ with the lower patch $2.5 \mathrm{~min}$ of arc closer than fixation and the upper patch $2.5 \mathrm{~min}$ of arc further away (see figure $1 \mathrm{~b}$ ). The baseline stimulus then reappeared and the subject pressed a key when the depths of the two patches again appeared to be equal and in the same plane as the fixation stimulus. The latency of the key press was recorded by the computer and a new $15 \mathrm{~s}$ baseline period began. Six cycles of adaptation, test, and recovery were given and the six reaction times were averaged to give the duration of the depth aftereffect. Alternate adaptation periods utilised upper-near and lower-near patches. During baseline and adaptation, the stereograms were redrawn once per second to prevent retinal afterimage formation.

For motion aftereffects the procedure was the same, except that the patches remained in the plane of fixation throughout. During the adaptation phases the dots in the upper patch drifted left or right at $5.5 \mathrm{deg} \mathrm{s}{ }^{-1}$, while those in the lower patch drifted in the opposite direction at the same velocity. These directions were reversed for alternate adaptation periods. The subjects pressed a key during the test periods when the relative apparent motion of the patches had ceased. 
During the adaptation phases, the fixation ' $\mathrm{X}$ ' was replaced by a stream of letters and numbers in random order, either at $4 \mathrm{~Hz}$ or $8 \mathrm{~Hz}$. In the control conditions, subjects ignored these numbers. In the attentional conditions, they pressed a key every time they saw a figure ' 8 ' or a figure ' 0 '; these comprised on average $17 \%$ of the characters presented (the distractors were A, H, I, M, T, U, V, W, X, and Y). These conditions were presented in counterbalanced order.

Six subjects engaged in the experiment, three of them naïve as to its purpose. Three did the motion aftereffect conditions first followed by the depth aftereffect, and three did them in the reverse order.

\section{Results}

In the control conditions, durations were within the range normally reported in the literature for these types of task. The durations were similar for the two aftereffects $(t=0.76)$. Although we could not calculate exact $d^{\prime}$ values for character detection in the secondary tasks, there were highly significant $(p<0.001)$ correlations between the numbers of button presses and appearances of the target figures ' 8 ' and ' 0 ', showing that attention was directed consistently towards the character stream during these phases. These correlations were lower with the $8 \mathrm{~Hz}$ character stream $\left(r_{34}=0.54\right.$ and 0.72 for depth and motion respectively) than with the $4 \mathrm{~Hz}$ stream $\left(r_{34}=0.74\right.$ and 0.96$)$, verifying that the faster condition was more difficult.

The pattern of aftereffect durations across conditions was consistent across stimulus domains (motion and depth), with aftereffect durations declining as the attentional task was introduced and as presentation rate was made faster. The results are shown in figure 2. Dark columns represent the no-response condition, light-grey represent the medium rate of response $(4 \mathrm{~Hz})$ and the white columns represent the difficult task $(8 \mathrm{~Hz})$. For both motion and depth aftereffects there was a progressive decline in aftereffect duration with attentional distraction from the adapting stimuli. A two-way analysis of variance with repeated measures reveals no significant difference between motion and depth durations $(F=0.03)$, but a highly significant effect of attentional condition $\left(F_{2,10}=12.69, p=0.002\right)$. Although the effect of attention was greater for depth (the aftereffect declined to $57 \%$ of baseline with the $8 \mathrm{~Hz}$ task, compared to $72 \%$ for motion), the interaction term is not significant $\left(F_{2,10}=1.84, p=0.209\right)$.

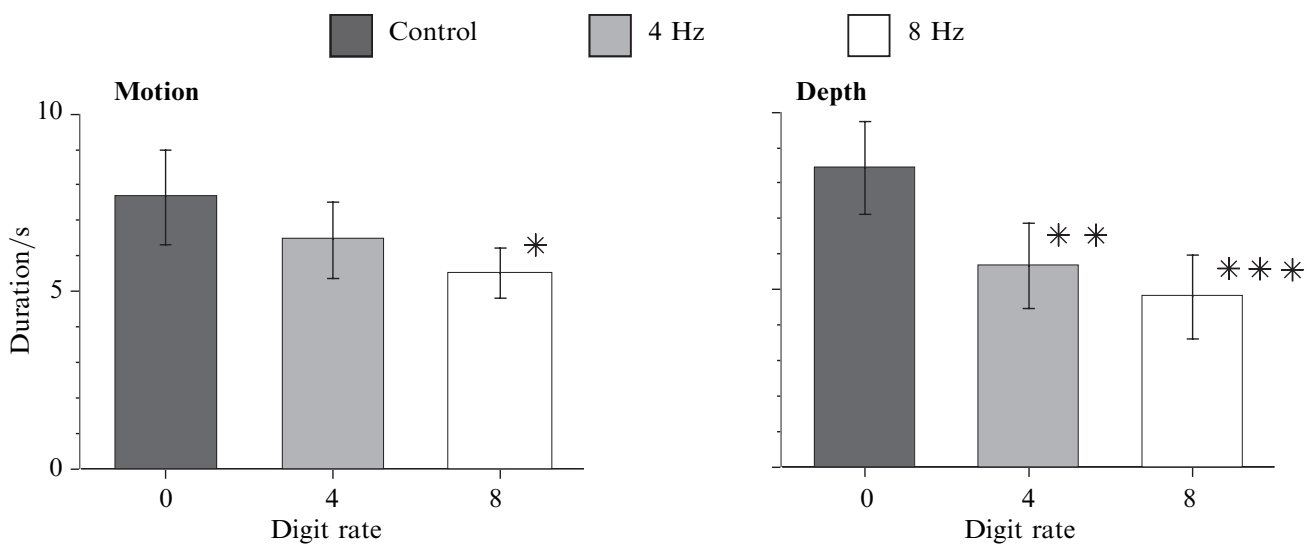

Figure 2. Motion (left) and depth (right) aftereffect durations, averaged across subjects. Dark columns show the control condition (no responses required), light-grey the medium distractor task (4 Hz character presentation), and white the faster task (8 Hz). Bars show standard errors. $*=p<0.05, * *=p<0.02, * * *=p<0.002, t$-tests relative to the control condition. 


\section{Discussion}

Our results show that attentional distraction modifies the visual mechanisms of depth perception. Moreover the magnitude of the effect was found to be quantitatively comparable to the effect on motion perception. The results thus extend to the depth domain Chaudhuri's (1990) finding of attentional modulation of motion perception and therefore add to the evidence refuting the inviolability of preattentive processing. Instead, they are in accord with the predictions of dynamic-feedback models of vision and attention.

Thus, the results support recent theories that 'early' channels are subject to feedback modulation from higher centres, not just for pattern recognition (Rose and Price 1995; Ullman 1995; Lee et al 1998; Pollen 1999; Rao and Ballard 1999; Friston and Price 2001) but as part of the mechanisms of selective attention (Deco and Schürmann 2000; Lamme and Roelfsema 2000; Lamme and Spekreijse 2000; Spivey and Spirn 2000; Suder and Wörgötter 2000; Schroeder et al 2001). Although psychophysical studies do not by themselves establish unequivocally the anatomical location at which the modulation occurs, recent fMRI evidence shows that top-down attention can enhance activity in the same areas that are activated by a complex image (Kastner et al 1999, page 757) and several studies have also demonstrated attentional effects on V1 neurophysiologically (Rees et al 1997; Büchel et al 1998; Watanabe et al 1998a, 1998b; Posner and Gilbert 1999; Smith et al 2000; Martínez et al 2001; Olson et al 2001). Our results are at one with another psychophysical demonstration that monocular channels are accessible to attention, by using stationary test patterns to assess the motion aftereffect in a Chaudhuri task (Nishida and Ashida 2000). Our data do not establish whether the change in the depth aftereffect also involves changes in monocular channels or in the mechanisms of stereopsis per se. Whichever is the case, both psychophysical studies imply the 'cognitive penetrability' of very early loci in the visual system.

The experimental method used here was to measure the strengths of the motion and depth aftereffects by determining their durations. This method is one of a range of possible techniques found in the literature, and was adopted here principally to allow us to keep the design of the experiments (stimuli and methods) clearly and directly related to the earlier studies of Blakemore and Julesz (1971) and of Chaudhuri (1990). A possible criticism of the use of duration measures is that the secondary attentional task might induce a criterion shift in judging the end of the aftereffect. Such a view was rejected for the motion aftereffect by Georgiades and Harris (2000b), who found that the secondary task induced changes in the apparent velocity of motion aftereffects, and in their rate of decay, as well as changes in their duration. The high correlation between the initial velocity and duration measures permitted Georgiades and Harris $(2000 \mathrm{a}, 2002)$ to continue using the duration measure only. Criterion shifts also cannot explain the effects of attentional manipulations on motion aftereffects assessed by nulling (Lankheet and Verstraten 1995) or by judging the direction of the aftereffect (Shulman 1993; von Grünau et al 1998; Alais and Blake 1999) in a test stimulus presented for a limited time. Although these studies were all conducted in the motion domain we reasoned that similar principles should apply also in the depth domain. In our experiments, we sought to make the criteria as sharp and reproducible as possible, which we did by using a display with two target squares embedded in a static background, wherein opposite aftereffects were expressed in the two target squares simultaneously, and the subject had to judge when their relative motion or depth had reached zero.

In conclusion, the results reported here provide direct psychophysical evidence that attention can modify the visual mechanisms of depth perception. This extends Chaudhuri's (1990) findings in the motion domain, and adds to the evidence against the autonomy of preattentive processing. 


\section{References}

Alais D, Blake R, 1999 "Neural strength of visual attention gauged by motion adaptation" Nature Neuroscience 21015 - 1018

Blakemore C, Julesz B, 1971 "Stereoscopic depth aftereffect produced without monocular cues" Science $171286-288$

Bradshaw M F, Rose D, 2002 "Attention, vision and action" Spatial Vision 15 115-116

Büchel C, Josephs O, Rees G, Turner R, Frith C D, Friston K J, 1998 "The functional anatomy of attention to visual motion. A functional MRI study" Brain $1211281-1294$

Cameron E L, Tai J C, Carrasco M, 2002 "Covert attention affects the psychometric function of contrast sensitivity" Vision Research $42949-967$

Chaudhuri A, 1990 "Modulation of the motion aftereffect by selective attention" Nature 344 60-62

Culham J C, Cavanagh P, 1994 "Motion capture of luminance stimuli by equiluminous color gratings and by attentive tracking" Vision Research $342701-2706$

Deco G, Schürmann B, 2000 "A hierarchical neural system with attentional top-down enhancement of the spatial resolution for object recognition" Vision Research 40 2845-2859

Driver J, Frackowiak R S J, 2001 "Neurobiological measures of human selective attention" Neuropsychologia $391257-1262$

Friston K J, Price C J, 2001 "Dynamic representations and generative models of brain function" Brain Research Bulletin $\mathbf{5 4} 275-285$

Georgiades M S, Harris J P, 2000a "The spatial spread of attentional modulation of the motion aftereffect" Perception 291185 - 1201

Georgiades M S, Harris J P, 2000b "Attentional diversion during adaptation affects the velocity as well as the duration of motion after-effects" Proceedings of the Royal Society of London, Series B $2672559-2565$

Georgiades M S, Harris J P, 2002 "Effects of attentional modulation of a stationary surround in adaptation to motion" Perception $31393-408$

Gray R, 2000 "Attentional modulation of motion-in-depth processing" Vision Research 40 $1041-1050$

Grünau M W von, Bertone A, Pakneshan P, 1998 "Attentional selection of motion states" Spatial Vision $11329-347$

Heydt R von der, Zhou H, Friedman H S, 2000 "Representation of stereoscopic edges in monkey visual cortex" Vision Research 401955 - 1967

Ho C E, 1998 "Letter recognition reveals pathways of second-order and third-order motion" Proceedings of the National Academy of Sciences of the USA $95400-404$

Kastner S, Pinsk M A, De Weerd P, Desimone R, Ungerleider L G, 1999 "Increased activity in human visual cortex during directed attention in the absence of visual stimulation" Neuron $22751-761$

Krauzlis R J, Adler S A, 2001 "Effects of directional expectations on motion perception and pursuit eye movements" Visual Neuroscience 18365 - 376

Lamme V A F, Roelfsema P R, 2000 "The distinct modes of vision offered by feedforward and recurrent processing" Trends in Neurosciences $23571-579$

Lamme V A F, Spekreijse H, 2000 "Modulations of primary visual cortex activity representing attentive and conscious scene perception" Frontiers in Bioscience 5 d232-243

Lankheet M J M, Verstraten F A J, 1995 "Attentional modulation of adaptation to two-component transparent motion" Vision Research $351401-1412$

Lee T S, Mumford D, Romero R, Lamme V A F, 1998 "The role of the primary visual cortex in higher level vision" Vision Research $382429-2454$

Martínez A, DiRusso F, Anllo-Vento L, Sereno M I, Buxton R B, Hillyard S A, 2001 "Putting spatial attention on the map: timing and localization of stimulus selection processes in striate and extrastriate visual areas" Vision Research $411437-1457$

Mehta A D, Ulbert I, Schroeder C E, 2000a "Intermodal selective attention in monkeys. I. Distribution and timing of effects across visual areas" Cerebral Cortex $10343-358$

Mehta A D, Ulbert I, Schroeder C E, 2000b "Intermodal selective attention in monkeys. II. Physiological mechanisms of modulation" Cerebral Cortex $10359-370$

Motter B C, 1993 "Focal attention produces spatially selective processing in visual cortical areas V1, V2, and V4 in the presence of competing stimuli" Journal of Neurophysiology 70 909-919

Nishida S, Ashida H, 2000 "A hierarchical structure of motion system revealed by interocular transfer of flicker motion aftereffects" Vision Research $40265-278$

Olson I, Chun M M, Allison T, 2001 "Contextual guidance of attention. Human intracranial event-related potential evidence for feedback modulation in anatomically early, temporally late stages of visual processing" Brain 124 1417-1425 
Paradiso M A, 2002 "Perceptual and neuronal correspondence in primary visual cortex" Current Opinion in Neurobiology $12155-161$

Plainis S, Murray I J, Chauhan K, 2001 "Raised visual detection thresholds depend on the level of complexity of cognitive foveal loading" Perception $301203-1212$

Poggio G F, 1995 "Stereoscopic processing in monkey visual cortex: a review", in Early Vision and Beyond Ed. T V Papathomas (Cambridge, MA: MIT Press) pp $43-53$

Pollen D A, 1999 "On the neural correlates of visual perception" Cerebral Cortex 9 4- 19

Posner M I, Gilbert C D, 1999 "Attention and primary visual cortex" Proceedings of the National Academy of Sciences of the USA $962585-2587$

Prince S J, Pointon A D, Cumming B G, Parker A J, 2000 "The precision of single neuron responses in cortical area V1 during stereoscopic depth judgements" Journal of Neuroscience $203387-3400$

Rao R P N, Ballard D H, 1999 "Predictive coding in the visual cortex: a functional interpretation of some extra-classical receptive-field effects" Nature Neuroscience 2 79-87

Raymond J E, 2000 "Attentional modulation of visual motion perception" Trends in Cognitive Sciences $442-50$

Rees G, Frith C D, Lavie N, 1997 "Modulating irrelevant motion perception by varying attentional load in an unrelated task" Science 278 1616-1619

Rose D, Price E, 1995 "Functional separation of global and local stereopsis investigated by cross-adaptation" Neuropsychologia $33269-274$

Schroeder C E, Mehta A D, Foxe J J, 2001 "Determinants and mechanisms of attentional modulation of neural processing" Frontiers in Bioscience 6 d672-684

Shulman G L, 1993 "Attentional effects on adaptation of rotary motion in the plane" Perception 22 947-961

Smith A T, Singh K D, Greenlee M W, 2000 "Attentional suppression of activity in the human visual cortex" NeuroReport $11271-277$

Spekreijse H (Ed.), 2000 "Pre-attentive and attentive mechanisms in vision. Perceptual organization and dysfunction" Vision Research $401179-1638$

Spivey M J, Spirn M J, 2000 "Selective visual attention modulates the direct tilt aftereffect" Perception \& Psychophysics $621525-1533$

Suder K, Wörgötter F, 2000 "The control of low-level information flow in the visual system" Reviews in the Neurosciences $11127-146$

Trotter Y, Celebrini S, Stricanne B, Thorpe S, Imbert M, 1996 "Neural processing of stereopsis as a function of viewing distance in primate visual cortical area V1" Journal of Neurophysiology $762872-2885$

Ullman S, 1995 "Sequence seeking and counter streams: a computational model for bidirectional information flow in the visual cortex" Cerebral Cortex 5 1-11

Vanduffel W, Tootell R B H, Orban G A, 2000 "Attention-dependent suppression of metabolic activity in the early stages of the macaque visual system" Cerebral Cortex 10 109-126

Vidyasagar T R, 1998 "Gating of neuronal responses in macaque primary visual cortex by an attentional spotlight" Neuro Report 91947 - 1952

Watanabe K, Shimojo S, 1998 "Attentional modulation in perception of visual motion events" Perception 271041 - 1054

Watanabe T, Harner A M, Miayuchi S, Sasaki Y, Nielsen M, Palomo D, Mukai I, 1998b “Taskdependent influences of attention on the activation of human primary visual cortex" Proceedings of the National Academy of Sciences of the USA $9511489-11492$

Watanabe T, Sasaki Y, Miyauchi S, Putz B, Fujimaki N, Nielsen M, Takino R, Mayakawa S, 1998a "Attention-regulated activity in human primary visual cortex" Journal of Neurophysiology 792218 - 2221

Yeshurun Y, Carrasco M, 2000 "The locus of attentional effects in texture segmentation" Nature Neuroscience 3 622-627 


\section{PERCEPTION}

VOLUME 322003

www.perceptionweb.com

Conditions of use. This article may be downloaded from the Perception website for personal research by members of subscribing organisations. Authors are entitled to distribute their own article (in printed form or by e-mail) to up to 50 people. This PDF may not be placed on any website (or other online distribution system) without permission of the publisher. 